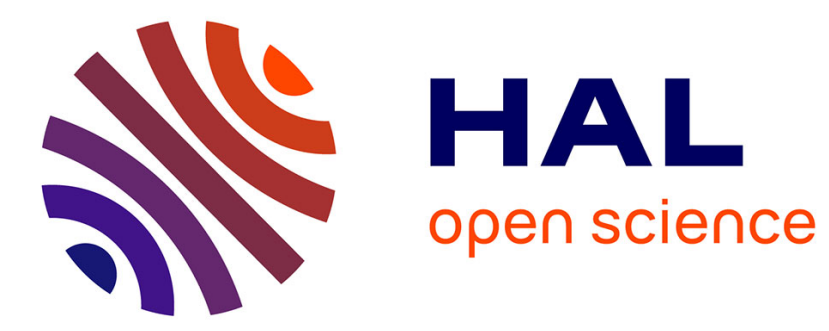

\title{
Jacques le fataliste : une ontologie spinoziste de l'écriture pluraliste \\ Yves Citton
}

\section{To cite this version:}

Yves Citton. Jacques le fataliste : une ontologie spinoziste de l'écriture pluraliste. Archives de Philosophie, 2008, Diderot Philosophe, pp.77-93. hal-00848110

\section{HAL Id: hal-00848110 https://hal.science/hal-00848110}

Submitted on 25 Jul 2013

HAL is a multi-disciplinary open access archive for the deposit and dissemination of scientific research documents, whether they are published or not. The documents may come from teaching and research institutions in France or abroad, or from public or private research centers.
L'archive ouverte pluridisciplinaire HAL, est destinée au dépôt et à la diffusion de documents scientifiques de niveau recherche, publiés ou non, émanant des établissements d'enseignement et de recherche français ou étrangers, des laboratoires publics ou privés. 


\title{
Jacques le fataliste: une ontologie spinoziste de l'écriture pluraliste
}

\author{
YVES CITTON
}

Université de Grenoble 3 Stendhal - umr LIRE

C'est sans doute partiellement par esprit de facétie que Denis Diderot en est arrivé à se faire appeler «le Philosophe " par ses amis, mais j’aimerais prendre cette appellation au pied de la lettre, et faire de lui l'auteur d'un des plus grands ouvrages de philosophie consacré au vieux problème de la liberté ontologique. Mon propos consistera en effet à soutenir ici que Jacques n'est pas un roman qui s'amuse à taquiner la philosophie, mais que ce texte éminemment littéraire constitue ce que la philosophie elle-même peut faire de mieux pour articuler philosophiquement le problème de la liberté humaine, en trahissant le moins possible son extrême complexité. De ce point de vue, qui semble partagé par les lectures les plus récentes de Jacques (celles proposées, entre autres, par Pierre Chartier ou Laurent Loty ${ }^{1}$ ), la forme romanesque et méta-fictionnelle qu'adopte la réflexion philosophique dans Jacques est la plus adéquate possible - plus adéquate que celle du traité philosophique - pour inviter les esprits humains à se faire une représentation pertinente de ce qu'est (et de ce que n'est pas) la liberté humaine. Dans la mesure où cette réflexion me paraît correspondre à la définition spinoziste de la liberté, je soutiendrai donc que le texte de Jacques rédigé par Diderot est, sur ce point au moins, plus authentiquement spinoziste que le texte de l'Éthique rédigé par Spinoza.

Il ne saurait être question de résumer ici en quoi consiste cette définition spinoziste de la liberté humaine. Il m'a fallu 580 pages dans mon dernier livre pour n'en repérer que les articulations, les présupposés et les impli-

1. Voir par exemple Pierre Chartier, Préface à Denis Diderot, Jacques le fataliste et son maître, Paris, Livre de Poche, 2000, p. 5-39 (c'est à cette excellente édition que nos références paginales renverront au cours de cet article), et Laurent LoTy, La Genèse de l'optimisme et du pessimisme (de Pierre Bayle à la Révolution française), Université de Tours, 1995. 
cations les plus générales ${ }^{2}$. Contentons-nous ici de l'évoquer à travers une analogie, celle que l'on peut établir entre le fatum du fataliste et les procédés d'écriture, et de souligner à partir de cette analogie ce qui semble distinguer le plus fortement le travail de Diderot de celui des autres écrivainspenseurs qui, autour de lui, contribuent à réinventer le spinozisme dans la France de la seconde moitié du XVIII ${ }^{\mathrm{e}}$ siècle - au premier rang desquels on peut penser au baron d'Holbach, qui met également en scène un «fataliste» dans son Système de la nature, ou à Léger-Marie Deschamps, qui développe une pensée apparemment très en phase avec celle de Diderot dans les années qui entourent leur rencontre de l'été 1769. La spécificité de Jacques (commencé en 1771), par rapport aux ouvrages de ces autres spinozistes, tient au double fait que son enjouement romanesque lui sert à la fois d'antidote à toute tendance dogmatique et de mise en relief du pluralisme inhérent à la sphère humaine de la substance unique spinoziste. Ces trois points seront passés en revue (écriture, anti-dogmatisme romanesque et pluralisme) à travers l'analyse rapide de l'épisode consacré par le récit à la machination du Père Hudson.

\section{Le spinozisme comme ontologie de l'écriture}

Les références explicites à Spinoza dans le texte de Jacques se réduisent à deux phrases discrètes mais cruciales. Dans la première, située au moment où se voit explicitée la philosophie spinoziste du valet, on apprend que «son capitaine lui avait fourré dans la tête toutes ces opinions qu'il avait puisées, lui, dans son Spinoza qu'il savait par cœur » (237). On perçoit la double ironie dont participe cette phrase: d'une part, cette adhésion au spinozisme est placée sous le registre de l'oü̈-dire, dont Spinoza lui-même fait emphatiquement, dans le Traité de la réforme de l'entendement, la forme la plus illusoire et la plus basse de connaissance; d'autre part, l'aspect mécanique et irréfléchi de cette reproduction par ouï-dire est accentué par les expressions choisies ("fourré dans la tête ", "savoir par cœur»), qui soulignent le caractère déterministe et conditionné de l'adhésion de Jacques aux formules mémorisées et répétitives de ce spinozisme. Si cette première occurrence du

2. Pour préciser la façon - méthodologiquement très problématique - dont j'utilise ici l'épithète "spinoziste ", voir Yves CitTon, L'Envers de la liberté. L'invention d'un imaginaire spinoziste dans la France des Lumières, Paris, éditions Amsterdam, 2006 (Introduction et chapitre I «L'épineuse contagion »). Sur la présence de la référence spinozienne dans la pensée des Lumières, voir les ouvrages classiques de Jonathan IsRAEL, Les Lumières radicales. La philosophie, Spinoza et la naissance de la modernité 1650-1750, Paris, éditions Amsterdam, 2005, et de Paul Vernière, Spinoza et la pensée française avant la Révolution, Paris, PUF, 1954. 
nom de Spinoza dans le texte du roman commence par dégrader la Raison de l'intellectualisme spinozien en une Réson irraisonnée se contentant de répéter la messe aux ânes, elle signale en même temps l'un des champs de réflexion qu'il convient de placer au cœur du spinozisme des Lumières, celui propre aux effets de résonance harmonique, dont Diderot avait fait le ressort principal du clavecin-philosophe évoqué par le Rêve de d'Alembert.

La seconde référence au philosophe hollandais intervient dans ce qui constituait la dernière phrase du récit (avant l'ajout d'un paragraphe supplémentaire dans le manuscrit destiné à Catherine II) qui voit Jacques épouser Denise, "avec laquelle il s'occupe à susciter des disciples à Zénon et à Spinoza, aimé de Desglands, chéri de son maître et adoré de sa femme, car c'est ainsi qu’il était écrit là-haut » (360). Ici encore, la référence à Spinoza s'ingénie à prendre son objet à contre-pied, puisque le nom de ce philosophe connu pour sa chasteté (et donc son absence de descendance biologique) sert de circonvolution pour évoquer l'acte sexuel reproducteur. Comme pour la première citation toutefois, ce contre-pied se retourne aussitôt en représentation adéquate du spinozisme, dans la mesure où le roman de Diderot, comme Jacques, s'occupe effectivement à susciter, non pas des rejetons biologiques, mais bien des "disciples » intellectuels à Zénon le stö̈que et à Baruch le fataliste.

Davantage que ce retournement d'un contre-pied ludique en une convergence plus profonde, retournement qui se répète dans ces deux évocations explicites du nom de Spinoza au sein du roman, ce qui paraît le plus profondément «spinoziste» dans Jacques, ce sont les résonances multiples du registre et du lexique de l'écriture à tous les différents niveaux du récit. C'est bien autour de cela que tournent discrètement les deux phrases où apparaît le nom du philosophe: la principale des formules apprises par cœur et répétées à toute occasion par le capitaine et le valet, c'est justement celle qui figure le fatalisme sous le forme de l' "écrit là-haut », qui scande le texte depuis la fin du premier paragraphe («Jacques disait que son capitaine disait que tout ce qui nous arrive de bien et de mal ici-bas était écrit là-haut », p. 43) jusqu'aux derniers mots de la (première) fin du texte («car c'est ainsi qu’il était écrit là-haut », p. 360).

Qu'une telle formule soit «spinoziste » ne saute certes pas aux yeux, puisqu'elle ne saurait figurer chez un penseur de l'immanence qui n'a de cesse de déboulonner toute forme de croyance en un quelconque «là-haut». Par quoi il s'avère déjà que le capitaine faisait bien plus que savoir et citer Spinoza "par cœur " puisque, comme tous les spinozistes des Lumières, il fait dire à l'auteur de l'Éthique ce que celui-ci n'a en réalité jamais dit lui- 
même... On comprend en quoi le "tout est écrit là-haut » est porteur des plus graves "trahisons " de l'orthodoxie spinozienne - et les défenseurs du dogme ne se sont pas privés de montrer à quel point les hommes du XVIII ${ }^{\mathrm{e}}$ siècle (Voltaire, Diderot, Jacques, son capitaine) ont mal compris et travesti la pensée «réelle» de Spinoza. Et pourtant, ici encore, cette bourde ludique qui tient en apparence du contre-sens provocateur fait en souterrain vibrer une corde discrète mais très sensible de la pensée spinoziste.

On ne peut vraiment mesurer la centralité de l'image de l'écriture dans la doctrine spinoziste que depuis la parution récente du beau livre que Lorenzo Vinciguerra a consacré à Spinoza et le signe. Résumons schématiquement l'approche que met patiemment et méthodiquement en place cette exégèse exemplaire du spinozisme. À partir des données minimales qu'esquisse furtivement la petite physique insérée après la proposition 13 de la deuxième partie de l'Ethique, Lorenzo Vinciguerra reconstitue une logique du traçage (c'est-à-dire de l'impression), fondée sur une tripartition entre le fluide, "qui, n'opposant pratiquement pas de résistance, ne retient quasiment pas les traces des corps extérieurs", le dur, défini " comme ce qui résiste le plus à la traçabilité, et donc aussi ce qui retient plus durablement les traces » et le mou, qui « est ce lieu moyen, entre le dur et le fluide, au sein duquel un corps est modifié par des autres, qui y laissent leurs traces »: " tout corps, dans la mesure où il est susceptible d'être un lieu de traces, c'est-àdire de porter les marques d'autres corps, peut être considéré comme étant plus ou moins mou » ${ }^{3}$. Une telle physique de la trace débouche sur une nouvelle définition du corps comme quelque chose qui "est écrit » et qui «écrit", définition au terme de laquelle «la constitution d'un corps, en tant qu'affection, n'est jamais que le résultat de ce que toutes les traces (innées et acquises) ont fait de lui »: "le corps ne doit pas être imaginé comme une portion d'étendue qu'il découperait par sa figure selon un dehors et un dedans, mais comme une certaine manière d'être affecté et d'affecter, c'est-à-dire comme une certaine manière d'être tracé, et une certaine manière de tracer l'étendue et de produire des signes" ${ }^{4}$.

C'est bien une ontologie de l'écriture que Lorenzo Vinciguerra exhume ainsi des textes de Spinoza. À la lumière de cette ontologie (spinoziste, dans la mesure où c'est des œuvres de ce philosophe que sont tirées les citations sur lesquelles se construit cette représentation du monde), la formule Tout

3. Lorenzo Vinciguerra, Spinoza et le signe. La genèse de l’imagination, Paris, Vrin, 2005, p. 129.

4. Lorenzo Vinciguerra, Spinoza et le signe, op. cit., p. 131 et p. 223. 
est écrit là-haut ne mérite pas tant d'être corrigée en substituant ici-bas à là-haut qu'elle ne demande à être précisée en un Tout s'écrit par traçage réciproque (quoique non égalitaire) - formule impliquant qu'une chose n'est que dans la mesure où elle est écrite et dans la mesure où elle écrit. Dès lors qu'on réfléchit au sein de cette conception plus large de l'écriture-traçage, les gribouillages intentionnels produits par les humains n'apparaissent plus que comme un registre très étroitement circonscrit d'un processus universel, dépassant de loin notre petit ici-bas, et s'étendant aussi bien dans le là-haut d'où viennent les rayons solaires qui marquent de rouge brûlant ou de bronzage aguichant le mou de notre épiderme:

Le mou du corps est le lieu où les corps s'inscrivent et s'écrivent en se signalant et se signifiant les uns aux autres. Les significations humaines sont une partie de ce processus infini. L'homme n'en est pas la source. [...] C'est à peine une métaphore de dire que le corps mou, quel qu'il soit (humain ou autre), est un espace d'écriture, qui vient nourrir ce que Baudelaire appelait «l'immense et compliqué palimpseste de la mémoire ». Sous la surface, d'autres écritures se conservent; d'autres stratifications et sédimentations de marques la travaillent. Le corps vit de ce rapport dynamique entre profondeur et surface. [...] Le corps est ainsi une écriture d'écritures, une mise en chaîne autant qu'une mise en scène de marques, qui s'enrichit et se complexifie avec l'expérience ${ }^{5}$.

Écrire, prescrire, contre-écrire, méta-écrire: l'acte-modèle du Père Hudson

En quoi, reconsidéré du point de vue d'une telle ontologie de l'écriture, Jacques le fataliste peut-il être dit "plus authentiquement spinoziste » que l'Éthique ou le Traité théologico-politique? Tentons de répondre à cette question à partir de l'analyse d'un épisode du roman dans lequel ce lexique de l'écriture - omniprésent dans l'ensemble de l'œuvre - apparaît avec une clarté presque «didactique». Dans le récit que le marquis des Arcis donne de la vie de son secrétaire et compagnon de route Richard, on se souvient de l'histoire de la contre-machination agencée par le père Hudson. Cet abbé, qui soumettait sa communauté de prémontrés à la plus grande austérité, tout en s'adonnant lui-même en secret à tous les plaisirs de la chair, s'était fait beaucoup d'ennemis, spécialement du côté des jansénistes qu'il avait persécutés sans merci. Ayant reçu (1) plusieurs mémoires - première apparition de l'écrit dans cet épisode - s'accordant à dénoncer la mauvaise conduite du Père Hudson, le général de l'ordre des prémontrés envoya en secret deux jeu-

5. Lorenzo Vinciguerra, Spinoza et le signe, op. cit., p. 167-168. 
nes religieux (dont Richard) comme commissaires chargés de (2) «constater juridiquement " la " liste des forfaits» dont il se rendait coupable (243-244).

Ne s'étant aperçu que très tard de cette accumulation d'écritures destinées à le perdre, le père Hudson réagit en montant une stratégie remarquablement retorse de contre-écriture, qui passe par la prescription - le terme est répété trois fois en un bref paragraphe (245) et fait apparaître une troisième forme d'écriture. Il (3) «prescrit » donc à une jeune fille qu'il avait séduite de donner rendez-vous aux deux commissaires et de leur raconter la séduction dont elle avait fait l'objet de sa part, dans tous ses détails les plus sordides. Lors de la rencontre qui s'ensuit, tandis qu'elle racontait sa «malheureuse histoire ", qui n'était que "trop réelle », l’un des commissaires (4) «prenait des notes sur ses tablettes » (246). Il ne restait plus aux deux envoyés du général, pour conclure leur mission, qu'à rédiger ces notes au clair pour en (5) «dresser un acte », après quoi la pauvre victime de l'indigne abbé n'aurait plus qu’à (6) «signer sa déclaration ». Le piège de l'abbé était toutefois sur le point de se refermer sur eux: (7) «Prenez la plume, dit-il à la jeune fille, et donnez-leur rendez-vous dans l'endroit que je vais vous indiquer » (246).

Une fois la jeune femme et les deux religieux réunis dans la maison, peu recommandable, prescrite par le père Hudson, et alors que (8) l'« on commençait à verbaliser ", un autre commissaire, de police cette fois, fait irruption, auquel le père Hudson (9) avait "dicté son rôle »: « deux religieux en tête-à-tête avec une fille » «indécemment vêtue » en un lieu tout sauf honnête, il ne restait plus au commissaire qu'à (10) «verbaliser à son tour " sur le comportement des deux commissaires verbalisants, «et comme il n’y avait rien dans son procès-verbal que l'exposition pure et simple du fait, (11) les deux moines furent obligés de signer " (247). Même si d'autres gestes relevant de l'écriture s'ajoutent à ceux-ci, arrêtons cette série au onze notations qui se sont succédées jusqu'ici en un peu moins de cinq pages, et tirons-en des enseignements sur la façon dont Jacques le fataliste représente le geste d'écriture.

Cet épisode illustre en effet à merveille ce que représente le fait d' « écrire » ou de "tracer " pour la réinvention que propose Diderot de l'ontologie spinoziste de l'écriture. À un premier niveau, écrire, selon la logique du traçage reconstituée par Lorenzo Vinciguerra, cela consiste, pour n'importe quel être, à (a) faire impression sur un autre être (le soleil brûle ou bronze ma peau, mon voyage en avion altère la couche d'ozone, mes pleurs indiquent à un observateur que je suis triste, les corps de jeunes femmes s'introduisant de nuit dans la maison de l'abbé Hudson révèlent que ses mœurs peuvent ne pas être aussi austères que celles qu'il impose à ses moines), sans qu'il n'y ait la moindre volonté de communication derrière aucune de ces 
actions. À un deuxième niveau, plus conforme à la façon dont nous imaginons communément le geste scriptural, écrire, c'est (b) inscrire une trace avec le dessein d'influencer le comportement d'autrui dans une certaine direction déterminée (prescrire un ordre à un subordonné, envoyer un mémoire à une autorité supérieure pour dénoncer le comportement d'un confrère). Mais écrire, c'est aussi, à un troisième niveau, statistiquement plus rare mais largement sur-représenté dans l'épisode du père Hudson, (c) agencer le geste même d'écriture, qu'il soit accompli par soi-même ou par autrui, au sein d'une méta-structure stratégisée qui surajoute une couche de finalités supérieures aux finalités premières de l'inscription: dresser un acte juridique, signer une déclaration, verbaliser, mais aussi dicter son rôle à quelqu'un, toutes ces formes de traçage relèvent d'une méta-écriture dont la vertu principale n'est pas à chercher dans le contenu immédiat de l'inscription, mais dans une structure de niveau supérieur (juridique, théâtrale) qui relaie et détourne l'influence première en direction d'une influence seconde. C'est bien entendu au sein de ce troisième type de (méta-) écriture que prennent place les gestes relevant du mensonge, de la manipulation, du spectacle et de la fiction.

En plus de nous donner l'occasion de mieux repérer ce niveau particulier de méta-écriture, l'épisode du Père Hudson suggère aussi quatre des principes généraux qui le régissent. Il invite d'abord à admettre que (I) l'efficace principale des actes humains tient à leur valeur de geste: ce qui emporte la décision dans le conflit dont fait l'objet le Père Hudson n'est pas tant la façon dont il agit vraiment en lui-même (avec la contradiction entre la règle qu'il impose aux autres et sa propre vie déréglée), mais l'impression que ses comportements tracent dans l'esprit d'autrui. Cet épisode pousse ensuite à reconnaître que (II) l'acte proprement humain consiste moins à marquer directement la réalité matérielle qu'à mettre en scène la façon dont d'autres humains y inscrivent leur marque: comme Madame de la Pommeraye, le Père Hudson agit en faisant agir autrui, et plus précisément en lui faisant jouer un rôle dont il tire les ficelles (affectives).

C'est bien une théorie de l'action que Diderot loge au cœur des épisodes les plus fortement narrativisés de ce roman consacré à dissiper les illusions de la liberté: l'agent le plus efficace (c'est-à-dire, d'un point de vue spinoziste, l'agent le plus libre) apparaît non seulement comme un acteur, capable de contrôler ses gestes pour produire l'impression qui lui convient le mieux dans le regard de son spectateur (comme l'explicite le Paradoxe sur le comédien), mais surtout comme un dramaturge qui écrit par avance ce que se diront (plus ou moins librement) les agents dont il manipule et 
anticipe les comportements - tel est bien le sens supérieur de "l'écriture " dans ce contexte. Madame de la Pommeraye "écrit » les gestes et les répliques des mère et fille d'Aisnon dont elle a inventé les personnages, et elle « écrit » du même coup les surprises, les désirs, les frustrations et le destin du marquis des Arcis qui mord à l'hameçon qu'elle lui tend à travers les d'Aisnon. De la même façon, le père Hudson "écrit " par avance les notes du rapport que les commissaires inscrivent sur leurs tablettes, «l'acte» qu'ils croient dresser librement, le procès-verbal sur lequel les deux moines sont obligés de signer leur défaite et son triomphe. Plus généralement, qu'est-ce que parler à ses collègues, à ses amis, à ses élèves, à ses enfants, sinon s'efforcer d'« écrire " par avance - à savoir de conditionner - (une partie de) leurs comportements futurs dans le sens qui nous semble le plus convenable à leurs intérêts et/ou aux nôtres?

Ce que l'épisode du Père Hudson ajoute cependant à la splendide histoire de Madame de la Pommeraye - qui n'est que la présentation méthodique d'un modèle d'acte disséqué au ralenti -, c'est que, contrairement au rôle d'emprunt par lequel les d'Aisnon sont contraintes de cacher leur identité réelle, (III) les rôles qu'on cherche à mettre en scène seront joués de façon d'autant plus efficace que les acteurs seront mis en position de "ne rien dire qui ne soit vrai" (245). Le comble du génie de l'abbé est de pousser la jeune fille qu'il a séduite à jouer son propre rôle de fille séduite, ce qui rend ses gestes d'autant plus crédibles qu'ils sont susceptibles de recevoir une sincérité intérieure et une vérification extérieure. De ce point de vue, on peut construire le spinozisme aussi bien comme un intellectualisme réaliste (qui valorise l'accès à la vérité pour elle-même) que comme un utilitarisme pragmatique (qui ne valorise l'accès au vrai que comme le chemin le plus sûr pour satisfaire nos besoins et nos désirs). Sur le plan éthique en tous cas, la leçon de Diderot est claire: il ne vaut la peine de jouer le jeu de la vérité et de la vertu que dans la mesure où ce jeu paraît être, d'un point de vue empirique, celui où l'on a le plus à gagner. Pour «écrire par avance " - pour "prescrire " - nos dialogues à venir de la façon la plus judicieuse, mieux vaudrait donc, pour autant que cela soit possible, ne rien (faire) dire qui ne soit vrai; il faudrait concevoir les personnages de façon à rester aussi près que possible du caractère des acteurs en charge de les assumer. Ce jeu de la vérité ne demeure toutefois qu'un jeu (de comédien et de hasard), dont les règles peuvent être infléchies ou contournées en fonction de nécessités et d'utilités locales, sous le contrôle d'un principe de précaution et de prudence.

Enfin, la nature conflictuelle du contexte dans lequel se déroulent les actes mis en scène par Madame de la Pommeraye comme par le Père Hudson 
suggèrent que (IV) l'émancipation relève de stratégies visant à écrire les comportements futurs à un niveau de méta-écriture plus élevé que celui auquel opère l'ennemi. Qu'il s'agisse de la vengeance d'une femme jalouse ou de la parade à l'attaque d'un général janséniste, dans les deux cas l'acte humain est modélisé comme relevant de calculs stratégiques, et comme se structurant à partir de conflits entre machinations et contre-machinations. Si Laurent Bove a déjà bien montré que le spinozisme était une pensée éminemment stratégique ${ }^{6}$, Diderot pousse les conséquences de cette intuition dans deux directions également suggestives. D’une part, conformément au principe de base du déterminisme, selon lequel aucune action n'est « originelle », mais relève toujours d'une " réaction » à des causes conditionnantes, il fait sentir à son lecteur que toute écriture mérite d'être située au sein d'un certain conflit et d'y apparaître comme une contre-écriture. Les modèles d'actes fournis par Madame de la Pommeraye et le Père Hudson visent tous deux à contre-effectuer le comportement d'autrui (la dérive du marquis des Arcis vers l'indifférence et l'infidélité, l'attaque du général janséniste et de ses deux commissaires).

Les deux cas suggèrent d'autre part que cette contre-écriture n'est gagnante que dans la mesure où elle parvient à se donner le statut d'une méta-écriture: échapper aux souffrances et aux asservissements que nous prépare autrui (volontairement ou non), capturer les bienfaits dont il est porteur, cela implique de pouvoir sur-coder les signes qui émanent de lui. Les deux commissaires croient contrôler la fille séduite lorsqu'ils insèrent son récit autobiographique dans le cadre supérieur d'une action en justice intentée contre le Père Hudson; celui-ci triomphe à son tour des deux moines parce qu'il a su intégrer leur procès-verbal dans le cadre plus englobant d'un second procès-verbal (relevant d'un niveau d'organisation plus élevé) qu'il a fait rédiger par son propre commissaire.

\section{Quel là-haut?}

On se trouve ici au noyau de l'intuition fondamentale que Jacques le fataliste partage avec la démonstration méthodique de l'Éthique spinozienne: les deux textes ne dénoncent l'illusion d'une liberté donnée (censée être inhérente à la nature de l'homme et de son âme) qu'afin de promouvoir

6. Sur le rôle central joué par le concept de stratégie dans la pensée spinoziste, voir Laurent Bove, La Stratégie du conatus. Affirmation et résistance chez Spinoza, Paris, Vrin, 1996. 
la construction active d'une liberté conquise (sur l'inhospitalité de notre milieu naturel et social) par un effort d'élévation. Dans le traité de Spinoza, c'est l'effort d'élévation par la connaissance rationnelle qui permet de conclure le parcours argumentatif sur une cinquième partie consacrée à la liberté humaine (ou à la puissance de l'intellect); dans le roman de Diderot, c'est la capacité des personnages actifs (Jacques, Madame de la Pommeraye, le Père Hudson, le chevalier de Saint Ouin) à méta-écrire le comportement des personnages passifs (le maître, le marquis des Arcis, Richard) qui fournit le modèle de l'agir humain au sein d'un monde de machines conditionnées (et conditionnantes). Bien entendu, aucun de ces personnages actifs du roman de Diderot ne saurait illustrer le modèle du «Sage » que cherche à promouvoir l'Éthique: hormis Jacques lui-même, qui incarne ce modèle de façon remarquablement convaincante, parce que remarquablement humaine, ils mettent tous leur admirable intelligence au service d'affects explicitement condamnés par Spinoza (la jalousie vengeresse; l'avidité sexuelle; la soif de richesse). Même si leur fortune finit par retourner leur victoire apparente en une défaite réelle (le bonheur conjugal du marquis des Arcis, la mort du chevalier de Saint Ouin), leur machination leur donne bien "la haute main » sur leur adversaire, au sein du cadre qu'ils s'étaient fixé euxmêmes.

Du point de vue de ce mouvement d'émancipation et de prise de pouvoir, conçu sur le modèle de l'élévation (et de la domination), méta-écrire consiste donc à écrire plus haut (à un niveau supérieur de discours qui vient sur-coder les discours d'autrui). Ce qui compte vraiment dans les rapports inter-humains, c'est moins ce que je dis à celui auquel je m'adresse sur un pied d'égalité, que ce qui s'écrit «là-haut ", à savoir dans le scénario sur-codé où j'insère les gestes d'autrui, généralement à son insu. Comme Jacques au moment où il avoue avoir "prémédité » la chute de cheval de son maître en «préparant» la rupture des courroies de son étrier, Madame de la Pommeraye et le Père Hudson peuvent affirmer indifféremment - et de façon absolument pertinente - que ce qui est arrivé aux « marionnettes » et aux «polichinelles » qu'ils se savent être était écrit « là-haut » ou (c'est-à-dire, sive) « dans [leur] prévoyance» (352) (selon le modèle du Deus sive Natura).

Ramener toutes nos écritures à un ici-bas immanent ne ferait somme toute que masquer la stratification qui articule nos interactions sociales. Notre réalité n'est pas faite d'égaux, commerçant de plain-pied, mais de multiples niveaux de sur-codage au sein desquels il est crucial de repérer les hiérarchies et les rapports de force. Le là-haut ne doit donc pas être rayé de notre discours, en même temps que la Providence contre laquelle Spinoza 
et Diderot s'accordent à multiplier les dénonciations; il doit être redéfini comme une fonction (quantitative et qualitative) du degré de prévoyance de prudence - et du degré de puissance qui caractérise chaque forme de comportement. Le capitaine de Jacques avait donc raison de répéter "que tout ce qui nous arrive de bien et de mal ici-bas était écrit là-haut » (43). Ce làhaut désigne, par exemple, les plans de guerre du général au fil desquels les fantassins sont conduits à la boucherie; ou les projections financières du gestionnaire, au nom desquelles mon usine sera fermée d'un trait de plume pour renaître de l'autre côté de la planète; ou l'article consacré par un journaliste aux amours de Bill, en fonction duquel le discours médiatique d'un pays basculera dans la quête de sperme présidentiel sur la robe d'une jeune assistante ${ }^{7}$.

Si la forme romanesque de Jacques peut être dite plus authentiquement spinoziste que la démonstration géométrique de l'Éthique, c'est qu'elle met en œeuvre, dans le type d'interlocution qu'elle instaure avec son lecteur, cet entre-jeu de méta-écritures croisées et conflictuelles entre individus soumis à leurs affects, entre-jeu dont Spinoza nous invite lui-même à penser, dans son Traité politique, qu'il détermine le devenir des sociétés humaines. Alors que la forme adoptée par l'Éthique postule un automate spirituel programmé à ne marcher qu'au pas régulier de la raison (même si l'espace des scolies permet de réintroduire plus ou moins discrètement son inscription dans un entre-jeu affectif), Jacques le fataliste non seulement prend les humains tels qu'ils sont (des mixtes rationalo-affectifs plus souvent enclins à rire et à pleurer qu'à se livrer à la difficile et rare recherche des causes), mais il les insère explicitement et pratiquement dans un type concret d'interaction qui les éclaire sur les principes premiers des interactions humaines.

La méta-fictionalité de Jacques le fataliste constitue en effet une scénographie ${ }^{8}$ permettant à la fois de révéler et d'analyser le rôle que jouent les phénomènes de méta-écriture au sein des relations inter-humaines. En faisant constamment intervenir la figure de l'auteur et celle du lecteur, au sein

7. "Vous êtes aux contes d'amour pour toute nourriture depuis que vous existez, et vous ne vous en lassez point. L'on vous tient à ce régime et l'on vous y tiendra longtemps encore, hommes et femmes, grands et petits enfants, sans que vous vous en lassiez. En vérité, cela est merveilleux » (238).

8. Sur la notion de métafiction, voir Jean-Paul Sermain, Métafictions (1670-1730). La réflexivité dans la littérature d'imagination, Paris, Champion, 2002; sur celle de scénographie, voir Dominique Maingueneau, Le discours littéraire. Paratopie et scène d'énonciation, Paris, Armand Colin, 2004. 
de rapports conflictuels qui amènent les deux parties à expliciter les principes régissant leurs stratégies respectives, Diderot habitue à poser systématiquement la question de savoir qui est en train de méta-écrire notre comportement, et à quelles fins. Quel là-haut est en train de faire de moi sa marionnette et son polichinelle, tandis que j'ai la naïveté de croire agir librement? En même temps que je pleure au récit de la magnanimité finale du marquis des Arcis ou que je ris aux tours joués par Jacques à son maître, la dimension méta-fictionnelle du roman me permet de mieux comprendre les jeux de sur-codage à l'œuvre dans les traçages qui nous constituent (activement et passivement) - à commencer par ceux qui régissent, ici et maintenant, l'interaction livresque dans laquelle je suis engagé.

Si l'Éthique de Spinoza peut prétendre à un degré de consistance unique parmi les ouvrages de philosophie, en ce qu'elle théorise l'automate spirituel en s'adressant à cet automate sous la forme automatique d'une démonstration more geometrico, l'enjouement romanesque de Jacques le fataliste reformule cet agencement en lui permettant d'intégrer, dans sa forme comme dans son contenu, la dimension affective de l'automate spirituel, ainsi que son insertion dans un monde stratifié de méta-écritures conflictuelles.

\section{Inventer un monisme pluraliste}

Pour mieux comprendre le tour d'écrou (ascendant) que Diderot impose à la pensée spinoziste à travers Jacques le fataliste, il est utile d'éclairer son geste par une comparaison rapide avec la façon dont deux autres « spinozistes " ont déployé leur réflexion durant ce troisième quart du XVIII ${ }^{e}$ siècle. Je tiens ici pour acquis que le contenu des systèmes philosophiques esquissés par le baron d'Holbach et par Léger-Marie Deschamps recoupe dans ses lignes principales celui qu'exprime Diderot à travers Jacques le fataliste ou le Rêve de d'Alembert. En refusant conjointement l'idée de Providence, et en souscrivant globalement aux thèses énoncées dans l'Appendice de la première partie de l'Éthique, les trois écrivains s'efforcent de penser l'être comme un multiple non pré-ordonné, dont chaque partie ne peut toutefois être proprement conçue qu'au sein d'une totalité en perpétuelle reformation. S'accordant à rejeter le dualisme chrétien du corps et de l'âme, ainsi qu'à admettre qu'il n'y a pas d'effet sans cause et que l'ensemble des individus s'auto-organisent conflictuellement au sein de cet individu globalisant (non-finalisé) qu'est la Nature, ils ont tous à négocier une difficile réconciliation entre un principe de multiplicité (puisque l'unité de l'univers ne sau- 
rait relever d'aucune intelligence centralisatrice) et un principe moniste (puisqu'il n'y a qu'une Totalité naturelle, au sein de laquelle tout est lié).

Sans entrer dans les nuances qui peuvent distinguer ces trois penseurs du point de vue du contenu de leur doctrine, abordons le problème de cette conciliation entre pluralisme et monisme à partir de la forme qu'ils ont choisi de donner à leur discours. Hormis quelques excursions fugaces dans le domaine du conte philosophique, et malgré le profond dialogisme d'une œuvre toute entière dédiée à la réfutation des impostures religieuses, le baron d'Holbach rédige des traités philosophiques de forme monologale classique. Sa pensée du multiple et du chaos - remarquablement fine, précise et suggestive (malgré les accusations de lourdeur qui le poursuivent depuis deux siècles) - s'exprime à travers une forme discursive qui nie ce multiple et ce chaos en les soumettant à un flux bien ordonnancé émanant d'une voix unique, seule maîtresse d'une argumentation à tendance dogmatique.

Léger-Marie Deschamps affiche pour sa part ce dogmatisme sans la moindre fausse pudeur, puisqu'il prétend - deux siècles avant Lacan - être la Vérité qui parle par sa bouche ${ }^{9}$. Il prend la peine de développer explicitement une ontologie de l'écriture qui résonne intimement avec la reconstruction vinciguerrienne du spinozisme. De même que, pour Spinoza, un corps «n'est jamais que le résultat de ce que toutes les traces (innées et acquises) ont fait de lui ", c'est-à-dire "une certaine manière d'être tracé, et une certaine manière de tracer l'étendue et de produire des signes " ${ }^{10}$, de même pour Deschamps « un être n'est que l'action des autres êtres sur lui, et réciproquement son action sur eux ». Pour illustrer la façon dont "tout se compose mutuellement et sans cesse dans le tout ", Deschamps en arrive lui aussi à mettre en scène l'interaction exemplaire qu'entretiennent un auteur avec son lecteur par la médiation du livre: «me lire et m'entendre lire, c'est se composer de mon ouvrage, qui agit alors physiquement par les yeux ou par les oreilles sur les fibres du cerveau, et qui les monte à tel ou tel ton, selon les impressions qu'il fait sur elles " ${ }^{11}$. La notion de cri de la vérité exprime chez Deschamps le circuit expressif par lequel le réel s'autoproduit en trouvant de quoi résonner/raisonner dans la façon dont il trace les corps humains. Chaque fois que j'écris, c'est la Ratio universelle, entendue comme

9. Sur Léger-Marie Deschamps, voir le recueil d'articles publié par Éric PuISAIS (éd.), Léger-Marie Deschamps. Un philosophe entre Lumières et oubli, Paris, L'Harmattan, 2001, ainsi que l'étude devenue classique d'André RoBinet, Dom Deschamps, le maître des maîtres du soupçon, Paris, Seghers, 1974.

10. Lorenzo Vinciguerra, Spinoza et le signe, op. cit., p. 131 et p. 223.

11. Léger-Marie Deschamps, Euvres philosophiques, Paris, Vrin, 1993, p. 227, 345 et 385. 
proportion et rapport entre les parties composantes de la nature, qui s'exprime à travers les tâtonnements de la Ratio humaine; chaque fois qu'on me comprend, c'est que mes propos résonnent avec la Ratio des parties qui composent le corps (et l'intellect) de mon lecteur: «Ce n'est que parce que je suis au fond la vérité que la voilà développée, et ce n'est que parce que mes semblables la sont ainsi que moi, que ceux d'entre eux capables de me lire et de me comprendre diront après m'avoir lu, et compris, c'est elle, la chose est évidente» ${ }^{12}$.

On est ici à la fois très proche de la façon dont Diderot figure l'entre-jeu des résonances entre clavecins-philosophes (une autre façon de nommer l'automate spirituel spinozien), et très éloigné de la façon dont il agence son discours et son interaction avec le lecteur. On pourrait résumer sommairement la différence principale entre les deux écrivains en disant que Deschamps ne conjugue la vérité qu'au singulier alors que Diderot invite son lecteur à la conjuguer au pluriel. Le multiple de Deschamps se borne à exprimer le même « fond » de vérité (métaphysique) à travers une innombrable diversité de bouches. Il est symptomatique de voir l'auteur du Mot de l'énigme et de La vérité tirée du fond du puits - notons les singuliers - affirmer que cette vérité est produite, à travers le langage, par la multitude des locuteurs d'une langue: «si le langage est formé, jusqu'à un certain point, d'après le cri de la vérité, c'est que ce n'est pas tel ou tel homme qui décide le langage, mais une généralité d'hommes, et que c'est à une généralité d'hommes que le cri du vrai se fait et doit toujours se faire le plus entendre " ${ }^{13}$. L'affirmation même de cette multitude neutralise cependant toute possibilité de multiplicité effective en condamnant cette "généralité d'hommes " à se mette à l'unisson d'un unique "cri du vrai ».

La plus intéressante originalité de Diderot au sein de la tradition spinoziste du XVIII ${ }^{\mathrm{e}}$ siècle paraît être au contraire de cultiver, dans ses formes d'expression comme dans la logique profonde de sa pensée, un pluralisme aussi radical que déroutant. Certes «tout est lié » et «l'indépendance absolue d'un seul fait est incompatible avec l'idée de tout; et sans l'idée de tout, plus de philosophie » ${ }^{14}$, mais l'expression de ce qui nous rapproche du vrai ne saurait jamais être monologale, ni prendre la forme d'un unisson, ni même d'un accord des contraires. L'indécidabilité du rapport à la vérité entretenue par

12. Léger-Marie Deschamps, Euvres philosophiques, op. cit., p. 401.

13. Léger-Marie Deschamps, Euvres philosophiques, op. cit., p. 399.

14. Denis Diderot, Pensées sur l'interprétation de la nature (1753), in Oeuvres, Versini éd., Paris, Robert Laffont, «Bouquin », tome I, p. 564. 
les voix incompatibles de moi et de lui dans Le Neveu de Rameau (ou par celles de A. et de B. dans le Paradoxe sur le comédien, ou par celles du père, du fils et de la plupart de leurs interlocuteurs dans l'Entretien d'un père avec ses enfants), la mise à l'avant-scène du jeu des contre-écritures et des métaécritures dans le dispositif narratif et dans les histoires contées dans Jacques le fataliste (comme dans Les deux amis de Bourbonne), tout cela s'efforce de faire naître l'intellection d'une tension entre discours incompatibles, voire irréconciliables.

Diderot consacre toute son œuvre, depuis la Lettre sur les aveugles jusqu'à l'Apologie de Sénèque en passant par l'agencement des voix multiples de l'Encyclopédie, à redéfinir la potentia intellectus dont Spinoza faisait le ressort de la libertas humana sous la forme d'une intel-lectio, c'està-dire d'une lecture située entre deux discours contradictoires, comme pour cultiver l'inconfort d'un entre-deux chaises en charge de prévenir tout risque de somnolence dogmatique. Il ne faut donc nullement s'étonner que Jacques le fataliste ait pu si souvent et si longtemps être considéré comme une dénonciation du déterminisme d'obédience spinoziste. Pour autant qu'on isole le roman de toutes les résonances que la position soutenue par Jacques entretient avec d'autres textes (formellement "philosophiques») de l'auteur, il n'est aucunement absurde d'y lire une mise en lumière des problèmes posés par la doctrine fataliste. Ce roman est bien une critique du spinozisme en même temps qu'il constitue son expression la plus adéquate! Allons encore un pas plus loin: c'est dans la mesure même où il donne toute sa force à la contre-écriture du spinozisme qu'il constitue l'expression la plus adéquate d'un spinozisme avide de solliciter la puissance du pluralisme (qui est puissance du multiple et de l'hétérogène).

Le spinozisme de Deschamps se donne pour horizon socio-politique un "état de mœurs " dont l'homogénéité proprement "totalitaire " a de quoi faire frémir: c'est un monde sans livre (on les a tous brûlés), donc sans lecture interpellante ni lecteur à interpeller et à manipuler comme une marionnette; c'est un monde parfaitement immédiat et transparent à lui-même, un monde totalement fluide, donc sans possibilité de traçage ni de résistance. L'univers dans lequel fait entrer Jacques est au contraire un monde d'opacité (ni le marquis des Arcis ni Richard n'entrevoient le premier mot de la contre-écriture qui va les prendre à son piège), un monde de résistance (c'est pour défendre leur honneur et leur mode de vie que Madame de la Pommeraye et le Père Hudson entreprennent de réécrire le comportement de leurs ennemis), un monde de déformation (où un capitaine peut apprendre et répéter par cœur ce que Spinoza n'a jamais écrit), un monde dans 
lequel une méta-écriture inédite peut à tout moment venir sur-coder et reconfigurer drastiquement le destin des individus et des formes sociales qu'ils se donnent. Le monisme de Diderot, conforme au principe spinozien selon lequel "il n'existe dans la nature qu'une substance unique » ${ }^{15}$, implique une reconnaissance d'interdépendance et de solidarité entre les différentes parties de la nature, sans pour autant faire de l'unanimisme la visée de l'organisation sociale. L'ontologie imprimée en nous par Jacques le fataliste reconnaît au contraire à cette substance unique la forme pluraliste d'un entrecroisement d'écritures antagonistes, d'autant plus intel-ligentes et effectives qu'elles auront su re-coder les traces de leurs adversaires, sans toutefois jamais pouvoir espérer d'autres victoires que locales et provisoires tant la pluralité implique que «si une chose quelconque est donnée», alors est également donnée «une autre plus puissante, par laquelle la première peut être détruite» ${ }^{16}$.

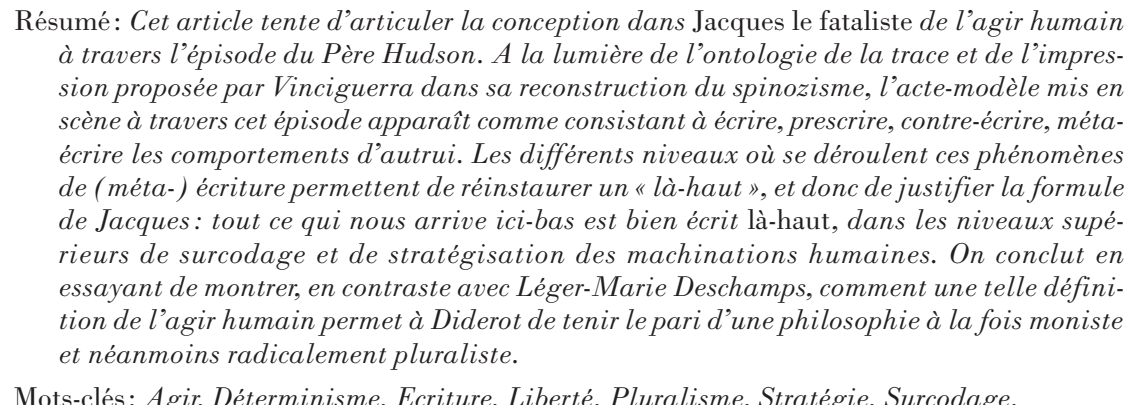

Mots-clés: Agir. Déterminisme. Ecriture. Liberté. Pluralisme. Stratégie. Surcodage.

Abstract:

Key words:

15. Spinoza, Éthique, partie I, proposition 10, scolie.

16. SpInoza, Éthique, partie IV, axiome. Pour les implications de cet axiome, qui constitue une source inépuisable de modestie, de prudence mais aussi d'espoir, voir le beau livre de Filippo Del Lucchese, Tumulti e indignatio. Conflitto, diritto e moltitudine in Machiavelli e Spinoza, Milano, Ghibli, 2004. 